\title{
ION-INDUCED ADSORPTION OF OXYGEN AT A Cu(110) SURFACE
}

\author{
A. G. J. DE WIT, J. M. FLUIT, TH. M. HUPKENS and R. P. N. BRONCKERS
}

Fysisch Laboratorium, Rijksuniversiteit Utrecht, Princetonplein 5, Utrecht. The Netherlands

\begin{abstract}
A preliminary report is given on experiments concerning the ion-induced adsorption of oxygen on copper. A strong enhancement of the sticking probability of oxygen is found during bombardment of the surface with neon ions in the keV region. The increase in the sticking probability is proportional to the primary ion intensities, used in this work $(0.25-10 \mu \mathrm{A} /$ $\mathrm{cm}^{2}$ ).
\end{abstract}

\section{Introduction}

The adsorption of oxygen at pressures between $10^{-8}$ and $10^{-4}$ torr on $\mathrm{Cu}(110)$ has already been investigated with LEED $^{1-4}$ ), work function measurements ${ }^{5}$ ) and ellipsometry combined with AES ${ }^{6}$ ). Some aspects of the adsorption of oxygen on $\mathrm{Cu}(110)$ surfaces were also studied with Low Energy Ion Scattering (LEIS). We determined the oxygen position on a $\mathrm{Cu}(110)$ surface with this techni$\left.q^{7} e^{7.8}\right)$. The results showed the oxygen atom to be lying between two neighbouring surface atoms in a $\langle 100\rangle$ row and $0.6 \AA$ below this row.

Studies of sputtering of oxygen adsorbates by low energy ions [ion-induced desorption ${ }^{9-11}$ )] showed that the amount of adsorbed species removed by the ions is a sensitive function of the energy of the ions in the $\mathrm{keV}$ region ${ }^{7.12}$ ).

In this paper we present experimental results, obtained with LEIS, concerning the adsorption of oxygen on a $\mathrm{Cu}(110)$ surface during ion bombardment. For the experimental methods we used a few assumptions are needed. (1) The residence time of the chemisorbed atoms is very long compared to the time scale of the experiments. (2) In signals related to the chemisorbed oxygen atoms there is hardly any contribution of physisorbed molecules; i.e. the coverage of the surface by physisorbed molecules is very low. (3) The measured ion signal $Y$ (ions scattered by oxygen) is proportional to the primary ion intensity $i_{0}$ (ions $/ \mathrm{cm}^{2} \mathrm{~s}$ ) and to the degree of coverage $\theta$ of chemisorbed oxygen atoms; i.e. $Y=C i_{0} \theta$, where $C$ contains collision cross sections and neutralization probabilities. To justify the third assumption our experiments were performed in such a way that all adsorbed oxygen atoms can act as scattering centres for the impinging noble gas ions; i.e. the beam direction is chosen such that the oxygen atoms are not shadowed by other neighbouring surface atoms.

\section{Experiments}

For the experiments we used a normal UHV system containing a twin parallel plate electrostatic analyzer to select the noble gas ions scattered by the oxygen atoms. The system is described in more detail elsewhere ${ }^{7,8,13}$ ). The partial oxygen pressure was measured with a quadrupole mass analyzer.

The target was mounted in a target manipulator after grinding and electro-lap-polishing. The surface was cleaned by sputtering with $\mathrm{Ne}^{+}$ions of a few $\mathrm{keV}$ at small elevation angles of incidence $\left(\varphi=20^{\circ}\right)$ until ion scattering energy spectra showed no contribution from impurity atoms (typically, applied ion dose $10^{16}$ ions $/ \mathrm{cm}^{2}$ ). After the cleaning procedure the energy spectra of scattered ions showed hardly any contributions of ion scattering from surface defects or crystal imperfections.

For the measurements the $\mathrm{Cu}(110)$ surface was bombarded with $4 \mathrm{keV} \mathrm{Ne}$ * ions at an elevation angle of incidence of $20^{\circ}$ in a $\langle 110\rangle$ surface direction. The detection angle was such that only a homogeneously bombarded part of the surface (i.e. homogeneous oxygen coverage) was observed by the analyzer.

\section{Experimental results}

The determination of the sticking probability $S$, without direct influence of the ion bombardment, was performed on target surfaces with an initial oxygen coverage resulting from an equilibrium between oxygen adsorption and ion-induced desorption. In a period of time $t$ during which the beam was switched of the oxygen coverage increased only due to spontaneous adsorption processes. After 
the time $t$ the ion beam was switched on again and the initial ion scattering signal $Y(t)\left(\sim i_{0} \theta\right)$ was recorded ${ }^{7,12}$ ). From these measurements the "spontaneous" sticking probability was determined with the equation

$S(\theta)=\frac{N_{\mathrm{s}}}{v} \frac{\mathrm{d} Y(t)}{\mathrm{d} t} \frac{\theta_{\max }}{Y_{\max }}$.

- $N_{\mathrm{s}}$ is the number of available chemisorption sites $/ \mathrm{cm}^{2}$ on an empty surface $\left[N_{\mathrm{s}}=1.08 \times 10^{15}\right.$ sites $/ \mathrm{cm}^{2}$ for $\mathrm{Cu}(110)$ ].

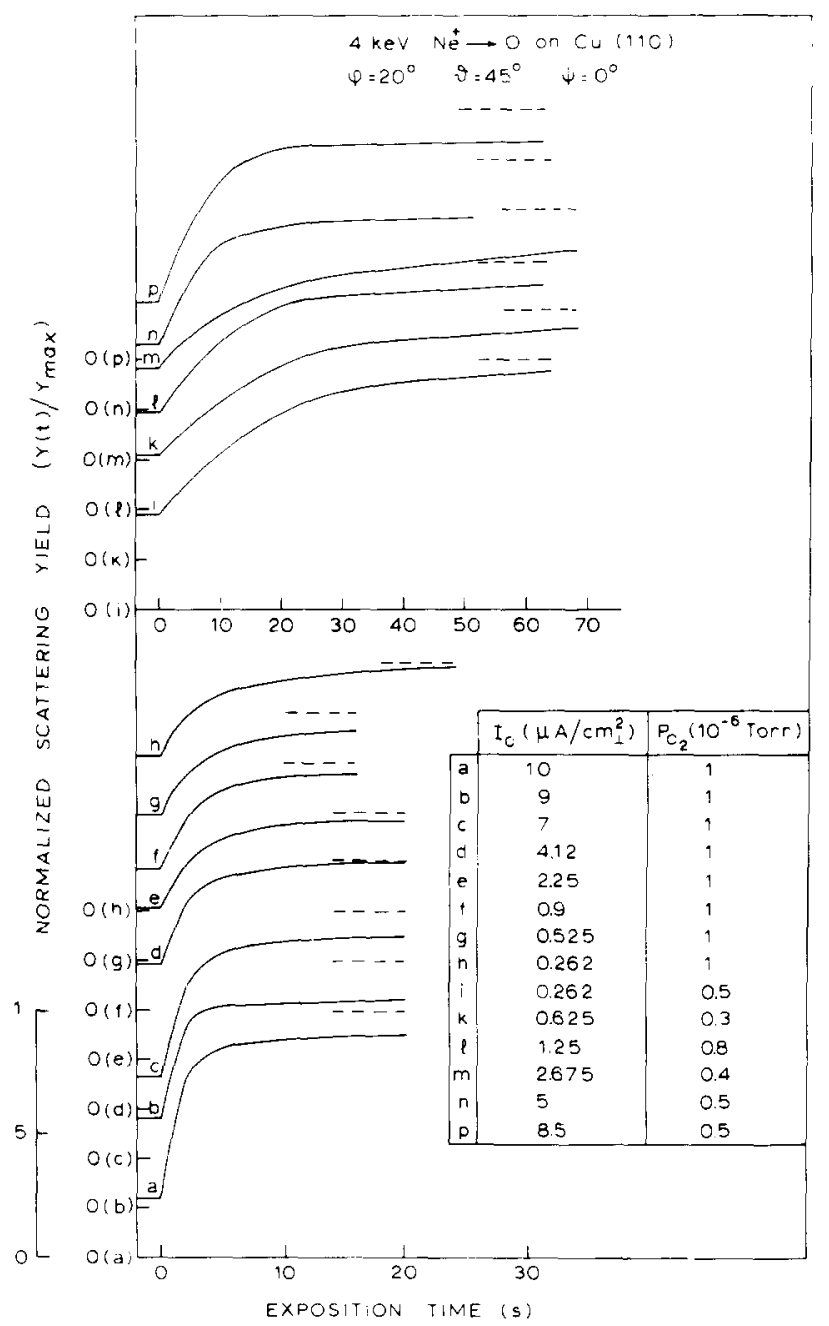

Fig. 1. Ion scattering signals of $4 \mathrm{keV} \mathrm{Ne}^{+}$ions scattered by $\mathrm{O}$ atoms on a $\mathrm{Cu}(110)$ surface as a function of exposition time. The different curves correspond to different primary ion intensities (and sometimes different oxygen pressures) which are presented in the insert. The zero-points of the different curves are shifted [indicated by $0(\mathrm{a}), 0$ (b), .. etc.]. The curves are normalized with respect to the maximum scattering signals (signal after $10 \mathrm{~min}$ exposure with ion beam off). Incident ion energies and experimental angles are given in the figure. $-v$ is the number of oxygen atoms (as molecules) hitting the surface (atoms $/ \mathrm{cm}^{2} \mathrm{~s}$ ) and

- $Y_{\max }$ is the measured ion signal at saturation coverage $\theta_{\max }$.

The results for $S$ are shown in fig. 2 (curve G). The values correspond reasonably with literature values $^{6}$ ) (when $\theta_{\max }=0.5$ is assumed).

The effect of ion-induced adsorption was found in experiments (with the ion beam on) where the oxygen pressure was suddenly increased until a certain value (about $10^{-6}$ torr). During this jump in oxygen pressure the ion scattering signal $[4 \mathrm{keV}$ $\mathrm{Ne}^{+} \rightarrow 0$ on $\left.\mathrm{Cu}(110)\right]$ was recorded as a function of time. The ion scattering signal increased initially due to dominating adsorption processes until, finally, an equilibrium was reached between the number of oxygen atoms being adsorbed and the number of oxygen atoms being desorbed (ioninduced desorption, sputtering). In fig. 1 the measured ion scattering signals are shown for different primary ion beam intensities indicated in the figure. The signals are divided by the signals $Y_{\max }$ measured at saturation coverage $\theta_{\max }$. The measurement of $Y_{\max }$ was performed by measuring the

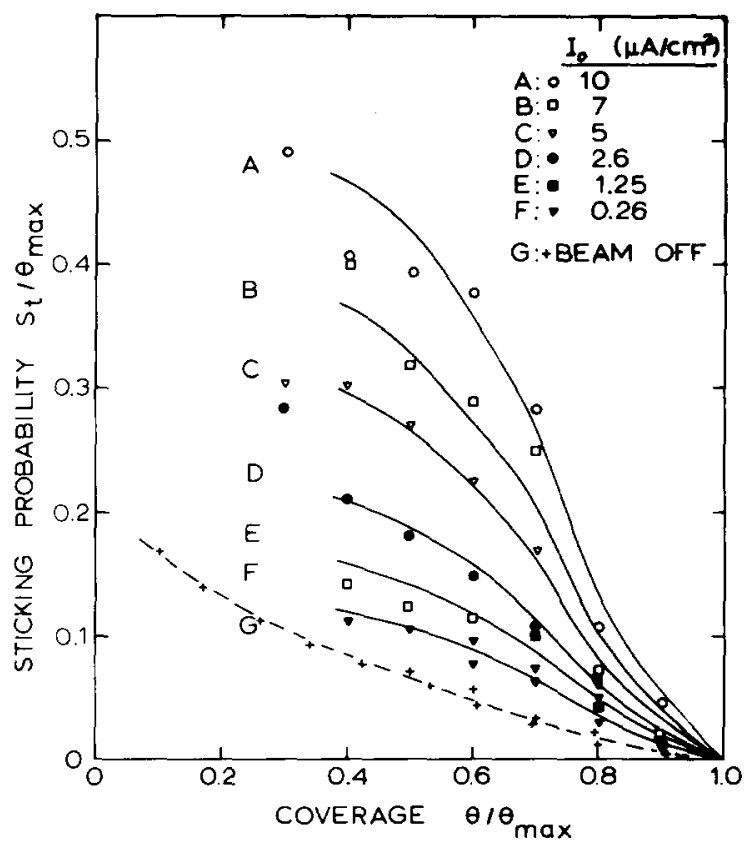

Fig. 2. The "normalized" sticking probability $S_{\mathfrak{t}} / \theta_{\max }$ of oxygen on a $\mathrm{Cu}(110)$ surface during $4 \mathrm{keV} \mathrm{Ne}{ }^{+}$ion bombardment at an incidence angle of $20^{\circ}$ (with the surface plane) as a function of the "normalized" coverage $\theta / \theta_{\max }\left(\theta_{\max }\right.$ is the saturation coverage). The different curves $(\mathrm{A}-\mathrm{F})$ correspond to different incident beam intensities $I_{0}$. Curve $G$ gives the sticking probability measured without the influence of the ion beam. 
initial ion scattering signal after a period of $10 \mathrm{~min}$ in which the ion beam was switched off. The values of $Y_{\max }$ varied proportionally to the used ion intensity, thus, obviously, $Y_{\max } \sim i_{0} \theta_{\max }$. The curves in fig. 1 show a steeper increase $\left[Y_{\max }^{-1}(\mathrm{~d} Y / \mathrm{d} t)_{t=0}\right]$ for higher ion beam intensities than for the lower intensities (at the same oxygen pressure). From these curves the sticking probability can be determined with the equation

$S_{\mathrm{t}}(\theta)=\frac{N_{\mathrm{s}}}{v}\left[\frac{\mathrm{d} Y(t)}{\mathrm{d} t}+Y(t) i_{0} \sigma\right] \frac{\theta_{\max }}{Y_{\max }}$.

$-i_{0}$ is the ion current intensity (ions $/ \mathrm{cm}^{2} \mathrm{~s}$ ), and

$-\sigma$ is the cross section for ion-induced desorption.

The results for $S_{\mathrm{t}}$ are shown in fig. 2 for different primary ion intensities. [In the calculations the earlier found ion-induced desorption cross section ${ }^{12}$ ) was used: $\sigma=8 \times 10^{-16} \mathrm{~cm}^{2}$. Note, that it has been assumed that the ion bombardment does not create chemisorption sites on the $\mathrm{Cu}(110)$ surface in which the oxygen atoms have a deviating position or a deviating binding]. At the largest ion intensity used $\left(10 \mu \mathrm{A} / \mathrm{cm}^{2}\right)$ the sticking probability appeared to be 4 times the "spontaneous" sticking probability. The relative increase of the sticking probability due to ion bombardment appeared to be nearly independent of $\theta$ : i.e. $S_{\mathrm{t}}(\theta)=S(\theta)\left(1+\lambda i_{0}\right)$, where $\lambda$ is a proportionality factor. In fig. 2 the best fits with this equation are shown also (drawn lines); for these fits $\lambda$ appeared to be $(5.5 \pm 1) \times 10^{-14} \mathrm{~cm}^{2} \mathrm{~s} /$ ion.

\section{Discussion}

To understand the phenomenon of ion-induced adsorption we have tried to estimate and/or to measure (1) the influence of fast ions (scattered and sputtered) on the oxygen molecules in the gas phase, and (2) the influence of surface damage on the adsorption process. It can be estimated that the influence of charged particles that cross the oxygen gas is only important (in our circumstances) for sticking probabilities in the order of $10^{-4}$ or lower ${ }^{7}$ ). For the possible influence of surface damage on the adsorption process, we found that immediately after a high dose of ion bombardment $\left(10^{17}\right.$ ions $\left./ \mathrm{cm}^{2}\right)$ the "spontaneous" sticking probability did not differ measurably from curve $G$ in fig. 2. Thus, one can conclude that only the presence of noble gas particles in or below the surface influences the adsorption process, in a way that the sticking probability increases with the number of fast particles impinging on the surface. However, the phenomenon itself needs to be investigated furthermore.

This work is part of the research programme of the Foundation for Fundamental Research on Matter (FOM) and was financially supported by the Dutch Organization for the Advancement of Pure Research (ZWO).

\section{References}

1) G. Ertl, Surface Sci. 6 (1967) 208.

2) G. W. Simmons. D. F. Mitchell and K. R. Lawless, Surface Sci. 8 (1967) 130.

3) A. Oustry, L. Lafourcade and A. Escaut, Surface Sci, 40 (1973) 545 .

4) N. Takahashi, H. Tomita and S. Motoo, Compt. Rend. 269B (1969) 618.

5) T. A. Delchar, Surface Sci. 27 (1971) 11.

6) F. H. P. M. Habraken and G. A. Bootsma, Surface Sci. 87 (1979) 333.

7) A. G. J. de Wit, Thesis, Utrecht, 1979.

8) A. G. J. de Wit, R. P. N. Bronckers and J. M. Fluit, Surface Sci. 82 (1979) 177.

9) E. Taglauer, G. Marin and W. Heiland, Appl. Phys. 13 (1977) 47.

10) E. Taglauer, G. Marin, W. Heiland and U. Beitat, Surface Sci. 63 (1977) 507.

11) E. Taglauer, U. Beitat and W. Heiland, Nucl. Instr, and Meth. 149 (1978) 605.

12) A. G. J. de Wit, R. P. N. Bronckers, Th. M. Hupkens and J. M. Fluit, Surface Sci., to be published.

13) A. v. Veen, A. G. J. de Wit, G. A. v. d. Schootbrugge and J. M. Fluit, J. Phys. E12 (1979) 861. 